\title{
Prevalência de inatividade física e apoio social dos pais em adolescentes de Macapá-Amapá
}

\author{
Prevalence of physical inactivity and parent's social support in adolescents \\ from Macapá-Amapá
}

\section{AUTORES \\ Danylo José Simões Costa ${ }^{1}$ (D) \\ Demilto Yamaguchi da Pureza ${ }^{2}(\mathbb{D})$ \\ Gregore Iven Mielke ${ }^{3,4}$ (C) \\ 1 Programa de Pós-Graduação em Ciências da Saúde, Universidade Federal do Amapá. Macapá, Amapá, Brasil. \\ 2 Programa de Pós-Graduacão em Ciências da Saúde, Universidade Federal do Amapá. Macapá, Amapá, Brasil. \\ 3 School of Human Movement and Nutrition Sciences, The University of Queensland, Brisbane, Australia. \\ 4 Programa de Pós-Graduação em Epidemiologia, Universidade Federal de Pelotas. Pelotas, Rio Grande do Sul, Brasil.}

\section{CONTATO}

Danylo José Simões Costa

danylo_costa18@hotmail.com

Programa de Pós-Graduação em Ciências da Saúde. Universidade Federal do Amapá. Rodovia JK S/N, Macapá, Amapá, Brasil. CEP: 68900-000.

DOI

10.12820/rbafs.v.22n6p533-39

\section{cc) $(9)$ BY NC ND}

Copyright: This is an open-access article distributed under the terms of the Creative Commons Attribution License ${ }^{\circledR}$, which permits unrestricted use, distribution, and reproduction in any medium, provided that the original author and source are credited.

\begin{abstract}
RESUMO
O estudo analisou a prevalência de inatividade física e a associação com o apoio social dos pais em adolescentes do ensino médio da área urbana de Macapá (AP). Trata-se de estudo transversal com amostra representativa de escolares de 14 a 19 anos de idade ( $n=1.205$ escolares), do ensino médio de escolas públicas e privadas. Os dados foram coletados por meio do Questionário para Medida de Fatores Associados à Atividade Física em Adolescentes para estimar a inatividade física $(<300$ $\mathrm{min} / \mathrm{sem}$ ) e o apoio social familiar. As demais variáveis foram: idade, sexo, cor da pele, idade, classe socioeconômica, ocupação e tipo de escola. A associação entre apoio social dos pais e inatividade física foi testada pela Regressão de Poisson com variância robusta. A prevalência de inatividade física foi de $60,2 \%$, sendo menor nos adolescentes do sexo masculino comparado ao feminino $(50,9 \%$ vs $68,0 \% ; \mathrm{p}=0,001)$. Os resultados da análise ajustada indicaram que a prevalência de inatividade física foi maior naqueles escolares cujo os pais nunca ou raramente: estimularam os filhos a fazer atividade física $(\mathrm{RP}=1,32$; IC95\%: 1,19-1,47), praticaram atividade física em conjunto com seus filhos $(\mathrm{RP}=$ 1,36; IC95\%: 1,18-1,57), transportavam/disponibilizavam transporte para a prática de atividade física $(R P=1,22$; IC95\%: 1,10-1,35), assistiam os filhos em atividades físicas $(R P=1,31$; IC95\%: 1,15-1,49), conversavam sobre atividade física ( $R P=1,20$; IC95\%: 1,08-1,33), elogiaram a prática de atividade física dos filhos $(\mathrm{RP}=1,34 ; \mathrm{IC} 95 \%: 1,20-1,50)$. Adolescentes que não recebiam apoio dos pais tendem a serem mais inativos do que seus pares que recebiam.
\end{abstract}

Palavras-chave: Inatividade física; Adolescentes; Apoio social. Estudo transversal.

\section{ABSTRACT}

This study analyzed the prevalence of physical inactivity and its association with parent's social support in high school adolescents living in the urban area of Macapa (AP). This is a cross-sectional study with a representative sample of 14- to 19-year-old adolescents $(n=1,205)$ from public and private high schools. Data were collected using the Questionnaire for Measurement of Factors Associated with Physical Activity in Adolescents to estimate physical inactivity ( $<300 \mathrm{~min} /$ week) and family social support. Additional variables included age, sex, skin color, age, socioeconomic position, occupation and type of school. The association between family social support and physical inactivity was analyzed using Poisson regression with robust variance. The prevalence of physical inactivity was $60.2 \%$, being lower in the male adolescents compared to the female $(50.9 \%$ vs $68.0 \%, p=0.001)$. The results of the adjusted analysis indicated that the prevalence of physical inactivity was higher in those students whose parents never or rarely: stimulated their children to perform physical activity (PR=1.32; 95\% CI: 1.19-1.47), they practiced physical activity together with their children ( $P R=1.36 ; 95 \%$ CI: 1.18-1.57), transporting / providing transport for the practice of physical activity $(P R=1.22$; 95\% CI: 1.10-1.35), watching your children perform physical activities $(R=1.31$, 95\% CI: 1.15-1.49), talked about physical activity ( $P R=1.20$; 95\% CI 1.08-1.33), praised their children's physical activity practice ( $P R=1.34 ; 95 \%$ CI: 1.20-1.50). Adolescents who did not receive parental support tended to be more inactive than their peers.

Keywords: Physical Inactivity; Adolescents; Social support.

\section{Introdução}

No Brasil, dados epidemiológicos de larga escala sobre inatividade física em crianças e adolescentes em todo o território nacional tem sido coletado na Pesquisa de Saúde do Escolar (PeNSE) ${ }^{1}$, mostrando que uma parcela importante dos adolescentes não atinge os níveis recomendados de atividade física ${ }^{1}$. Estudos realizados em outras localidades brasileiras, têm descrito prevalências de inatividade física em crianças e adolescentes tendo variações no ciclo de vida de 5,3\% a 94\% ${ }^{2}$. Entretanto, há poucos dados sobre inatividade física na região amazônica, norte do país. 
A inatividade física está entre os quatro principais fatores de riscos para mortalidade global, perdendo para a pressão arterial elevada, tabagismo e glicose sanguínea elevada, e superando o sobrepeso/obesidade ${ }^{3}$. Inúmeros fatores podem influenciar as altas proporções de inatividade física em adolescentes, incluindo indicadores sociodemográficos, econômicos e de apoio social/familiar.

Nesse contexto, a ausência de apoio social demandada a relativas participações a serem direcionadas por meio das redes sociais formadas pelo indivíduo ${ }^{4}$ pode indicar possíveis alterações futuramente na adoção de estilo de vida ativo influência pelo meio familiar. $\mathrm{O}$ apoio social para a prática de atividade física é um construto multidimensional, caracterizado por ações provenientes de diferentes fontes e pessoas como, por exemplo, a família e os amigos; podendo variar em diferentes tipos: levar, convidar, acompanhar, incentivar e elogiar o jovem para a realização de atividades físicas, como também acompanhá-lo e elogiá-lo nessas ações ${ }^{5}$.

Em estudo nacionais e internacionais tem sido observado que o fornecimento de apoio social dentro do ambiente familiar e dos amigos está associado a maiores níveis de atividade física em adolescentes ${ }^{6,7,8}$. No entanto, a associação entre o apoio social dos pais e a inatividade física pode variar de acordo com o contexto social e cultural no qual o adolescente se encontra. Embora esta associação já tenha sido testada em estudos brasileiros ${ }^{1,2,9,10}$, sobretudo nas regiões sul, sudeste e nordeste, há uma carência de estudos na região norte.

De acordo com este cenário, o presente estudo teve como objetivo analisar a prevalência de inatividade física e a associação com o apoio social dos pais e $\mathrm{m}$ adolescentes do ensino médio de escolas públicas e privadas da área urbana de Macapá (AP).

\section{Métodos}

Este estudo foi caracterizado como transversal de base escolar, e teve como objetivo principal, estimar a prevalência de escolares fisicamente inativos e o apoio social familiar. Os dados referentes à população de alunos foram disponibilizados pela Secretaria de Estado da Educação (SEED/AP), considerando faixa etária entre 14 a 19 anos, matriculados no ensino médio. A população da pesquisa foi estimada em 44.280 alunos matriculados no ensino médio na área urbana cidade Macapá (AP), na região norte do Brasil.

A amostragem foi selecionada por conglomerados em dois estágios (escolas e turmas). Todas as escolas da área urbana do município de Macapá foram consideradas elegíveis, sendo que no primeiro estágio, foram sorteadas 9 escolas, proporcionalmente distribuídas por tipo (6 públicas e 3 privadas). No segundo estágio, foram sorteadas 54 turmas, distribuídas de forma proporcional entre o $1^{\circ}$, $2^{\circ}$ e $3^{\circ}$ ano do ensino médio (diurno e vespertino).

$\mathrm{Na}$ coleta dos dados foi aplicado o questionário QFAAF (Questionário para Medida de Fatores Associados à Atividade Física em Adolescentes) ${ }^{10}$. Dentre as principais vantagens do QFAAF, destaca-se a sua capacidade em mensurar tempo, frequência e duração das atividades físicas praticadas e a possibilidade de realizar estimativas de atividade física segundo as recomendações atuais para adolescentes, bem como a facilidade de aplicação e de preenchimento por parte dos adolescentes, além de variáveis de apoio ou apoio social advindas dos pais ou responsáveis para adesão ou a prática de atividade física.

O questionário foi autopreenchido pelos alunos na própria sala de aula sendo feito de forma anônima, após leitura prévia do avaliador, de cada questão. O questionário foi preenchido no horário normal de aula, preferencialmente agendados durante as aulas de Educação Física na semana letiva, sendo o tempo médio para preenchimento de 15 minutos, em média. Nas escolas públicas pesquisadas a pesquisa aconteceu em dois períodos de funcionamento da escola, de manhã (das $7 \mathrm{~h}$ às 12h30), à tarde (das $13 \mathrm{~h} 30$ às 18h30), já nas escolas particulares as coletas aconteceram no turno da manhã (das 07:00 às 12:45).

As variáveis sociodemográficas mensuradas foram: sexo (masculino e feminino), cor da pele (branca, parda e negra), faixa etária (14-15, 16-17 e 18-19), classificação econômica (A, B, C e D/E), tipo de escola (pública e privada), ocupação (não e sim) escolaridade do pai (fundamental incompleto, fundamental completo, médio completo e superior completo), escolaridade da mãe (fundamental incompleto, fundamental completo, médio completo e superior completo) e ano do ensino médio ( $1^{\circ}$ ano, $2^{\circ}$ ano e $3^{\circ}$ ano). A classificação econômica dos adolescentes foi mensurada pelo questionário de classificação socioeconômica da Associação Brasileira de Empresas de Pesquisa (ABEP, 2003) ${ }^{11}$ que leva em consideração a escolaridade do chefe da família e alguns bens de consumo que a casa dos participantes possui, sendo a classificação subdivida em de A, B, C, D/E.

$\mathrm{Na}$ medida da prática de atividade física os adolescentes reportavam a frequência com que praticaram (dias/semana) e a duração (minutos/dia) das atividades físicas realizadas na semana anterior à participação na 
pesquisa, considerando uma lista contendo 24 atividades, com possibilidade de os adolescentes acrescentarem mais duas atividades. Para manter a comparabilidade com estimativas nacionais, foram classificados como inativos aqueles participantes que não atingiram 300 minutos semanais de atividade física na semana ${ }^{1}$.

$\mathrm{O}$ apoio social foi mensurado por questões sobre a frequência semanal com que os pais realizavam ações para facilitar ou incentivar a realização de atividades físicas, com quatro alternativas resposta para cada uma: nunca, raramente, frequentemente e sempre. Para efeitos de análise, as opções "nunca" e "raramente", bem como as opções "frequentemente" e "sempre", foram agrupadas, formando as categorias de apoio social dos pais "nunca/raramente" e "frequentemente/sempre", respectivamente. Para este estudo, os itens de apoio avaliados foram a frequência com que os pais: a) estimulam os filhos a praticarem atividade física; b) praticam atividade física com os filhos; c) transportam/disponibilizam transporte; d) assistem os filhos durante a atividade física; e) conversam com os filhos sobre atividade física; f) elogiam a prática das atividades físicas dos filhos.

Os dados coletados foram processados e checados duplamente no software Epi-Info 3.52, sendo convertidos para análise no pacote estatístico Stata 12. Inicialmente foi realizada análise descritiva com cálculo de prevalência de inatividade física em cada grupo das variáveis independentes. A associação ente a fatores sociodemográficos e a prevalência de inatividade física foi testada utilizando o teste do Qui-quadrado de heterogeneidade ou para tendência linear, de acordo com a natureza da variável de exposição. Num segundo momento foram realizadas análises brutas e ajustadas utilizando modelos de regressão de Poisson, com variância robusta. As análises ajustadas foram conduzidas incluindo no modelo final todas as variáveis associadas com a inatividade física com um valor $\mathrm{p}$ inferior a 0,20 na análise bruta. Para todos os testes foram adotados $5 \%$ de nível de significância.

O presente estudo foi aprovado pelo Comitê de Ética em Pesquisa da Universidade Federal do Amapá, sob o parecer número 34241014.6.0000.0003. Todos os adolescentes que participaram do estudo foram autorizados pelos pais ou responsáveis.

\section{Resultados}

Foram elegíveis para o estudo 1.310 escolares. Após as perdas e recusas a amostra estudada incluiu 1.205 escolares ( $\mathrm{n}=670 ; 55,4 \%$ do sexo feminino) com faixa etária entre 14 a 19 anos (média 15,6 anos $\pm 1,81$ ), de escolas públicas estaduais $(66,3 \%)$ e privadas $(33,7 \%)$ no município de Macapá, Amapá, Brasil. A maior parte dos jovens se autodeclararam pardos (49,0\%), em seguida bancos $(33,9 \%)$ e negros $(17,2 \%)$. Cerca de $37 \%$ pertenciam a classificação econômica $\mathrm{C}$ e $88 \%$ responderam que não possuíam qualquer tipo de ocupação com trabalho remunerado. Em relação à escolaridade dos pais, $43,2 \%$ e 50,4\% relataram escolaridade de nível superior para seu pai e mãe, respectivamente (Tabela 1).

Tabela 1 - Características sociodemográficas da amostra e prevalência de inatividade física e fatores associados em adolescentes da cidade de Macapá (Amapá), 2016, ( $n=1.205)$.

\begin{tabular}{|c|c|c|}
\hline Variáveis & $\mathrm{n}$ & $\%$ \\
\hline \multicolumn{3}{|l|}{ Sexo } \\
\hline Masculino & 534 & 44,6 \\
\hline Feminino & 664 & 55,4 \\
\hline \multicolumn{3}{|l|}{ Cor da pele } \\
\hline Branca & 407 & 33,9 \\
\hline Parda & 588 & 49,0 \\
\hline Negra & 206 & 17.2 \\
\hline \multicolumn{3}{|l|}{ Faixa etária } \\
\hline $14-15$ & 525 & 43,6 \\
\hline $16-17$ & 604 & 50,1 \\
\hline $18-19$ & 76 & 6,3 \\
\hline \multicolumn{3}{|l|}{ Classificação econômica } \\
\hline A & 91 & 7,6 \\
\hline $\mathrm{B}$ & 433 & 36,1 \\
\hline $\mathrm{C}$ & 452 & 37,7 \\
\hline $\mathrm{D} / \mathrm{E}$ & 224 & 18,7 \\
\hline \multicolumn{3}{|l|}{ Tipo de escola } \\
\hline Pública & 803 & 66,6 \\
\hline Privada & 402 & 34,4 \\
\hline \multicolumn{3}{|l|}{ Ocupação } \\
\hline Não & 1.053 & 87,8 \\
\hline Sim & 147 & 12,2 \\
\hline \multicolumn{3}{|l|}{ Escolaridade do pai } \\
\hline Fundamental incompleto & 240 & 19,9 \\
\hline Fundamental completo & 136 & 11,3 \\
\hline Médio completo & 308 & 25,6 \\
\hline Superior completo & 521 & 43,2 \\
\hline \multicolumn{3}{|l|}{ Escolaridade da mãe } \\
\hline Fundamental incompleto & 196 & 19,9 \\
\hline Fundamental completo & 90 & 11,3 \\
\hline Médio completo & 312 & 25,9 \\
\hline Superior completo & 607 & 50,4 \\
\hline \multicolumn{3}{|l|}{ Ano do ensino médio } \\
\hline $1^{\circ}$ ano & 426 & 35,4 \\
\hline $2^{\circ}$ ano & 515 & 42,7 \\
\hline $3^{\circ}$ ano & 264 & 21,9 \\
\hline \multicolumn{3}{|l|}{ Nível de atividade física } \\
\hline Ativo & 479 & 39,8 \\
\hline Inativo & 726 & 60,2 \\
\hline
\end{tabular}


A Tabela 2 apresenta a prevalência de inatividade física e sua associação com variáveis sociodemográficas. $\mathrm{Na}$ amostra total, 60,2\% (IC95\%: 57,4-63,0) dos estudantes não atingiram pelo menos 300 minutos por semana em atividades físicas. A prevalência de inatividade física foi maior no sexo feminino $(68,0 \%)$ do que no masculino $(50,9 \%, \mathrm{p}=0,001)$. A cor da pele $(\mathrm{p}=$ 0,714), a classificação econômica $(\mathrm{p}=0,565)$, o tipo de escola $(p=0,200)$ e a faixa etárias $(p=0,211)$ não se associaram à inatividade física. Foi possível verificar que a prevalência de inatividade física foi menor nos escolares que estavam em séries mais avançadas.

Tabela 2 - Prevalência de inatividade física e fatores associados em adolescentes de Macapá (Amapá), 2016, (n=1.205).

\begin{tabular}{|c|c|c|c|}
\hline \multirow{2}{*}{ Variáveis } & \multirow{2}{*}{$\begin{array}{c}\begin{array}{c}\text { Fisicamente } \\
\text { inativos }\end{array} \\
\mathrm{n}(\%)\end{array}$} & \multicolumn{2}{|c|}{ Análise bruta } \\
\hline & & RP (IC95\%) & $p^{*}$ \\
\hline Sexo & & & $<0,001$ \\
\hline Masculino & $272(50,9)$ & 1,00 & \\
\hline Feminino & $451(68,0)$ & $1,33(1,21-1,48)$ & \\
\hline Cor da pele & & & 0,714 \\
\hline Branca & $244(60,0)$ & 1,00 & \\
\hline Parda & $361(61,4)$ & $1,02(0,92-1,13)$ & \\
\hline Negra & $120(58,3)$ & $1.59(0,84-1,12)$ & \\
\hline Faixa etária & & & 0,441 \\
\hline $14-15$ & $327(62,3)$ & $0,94(0,86-1,04)$ & \\
\hline $16-17$ & $355(58,8)$ & $0,93(0,76-1,14)$ & \\
\hline $18-19$ & $44(57,9)$ & 1,00 & \\
\hline Classificação econômica & & & 0,565 \\
\hline A & $49(53,8)$ & 1,00 & \\
\hline B & $258(59,5)$ & $1,11(0,90-1,36)$ & \\
\hline $\mathrm{C}$ & $276(61,1)$ & $1,13(0,92-1,39)$ & \\
\hline $\mathrm{D} / \mathrm{E}$ & $140(62,5)$ & $1,16(0,94-1,44)$ & \\
\hline Tipo de escola & & & 0,211 \\
\hline Pública & $494(61,5)$ & 1,00 & \\
\hline Privada & $232(57,5)$ & $0,94(0,85-1,04)$ & \\
\hline Ocupação & & & 0,316 \\
\hline Não & $642(61,0)$ & 1,00 & \\
\hline Sim & $83(56,5)$ & $0,93(0,80-1,08)$ & \\
\hline Escolaridade do pai & & & 0,085 \\
\hline Fundamental incompleto & $151(62,9)$ & 1.00 & \\
\hline Fundamental completo & $86(63,9)$ & $1,01(0,86-1,18)$ & \\
\hline Médio completo & $197(64,0)$ & $1,02(0,89-1,16)$ & \\
\hline Superior completo & $292(56,1)$ & $0,89(0,79-1,01)$ & \\
\hline Escolaridade da mãe & & & 0,469 \\
\hline Fundamental incompleto & $125(63,8)$ & 1,00 & \\
\hline Fundamental completo & $56(62,2)$ & $0,98(0,80-1,18)$ & \\
\hline Médio completo & $192(61,5)$ & $0,96(0,84-1,11)$ & \\
\hline Superior completo & $353(58,2)$ & $0,91(0,80-1,04)$ & \\
\hline Ano ensino médio & & & 0,010 \\
\hline $1^{\circ}$ ano & $267(62,7)$ & 1,00 & \\
\hline $2^{\circ}$ ano & $323(62,7)$ & $1,00(0,91-1,10)$ & \\
\hline $3^{\circ}$ ano & $136(51,5)$ & $0,82(0,72-0,94)$ & \\
\hline
\end{tabular}

RP= Razão de prevalência; IC95\%= Intervalo de confiança de 95\%; *Qui-quadrado para tendência linear.
Verificou-se que em torno de $65 \%$ dos adolescentes relataram nunca/raramente receber algum tipo de apoio social dos pais (Tabela 3). De uma forma geral, a falta de apoio dos pais esteve associada com a maior prevalência de inatividade física nos adolescentes. A prevalência de inatividade física em adolescentes que nunca/raramente foram estimulados por seus pais a praticarem atividade física foi 32\% maior quando comparados aos seus pares $(\mathrm{RP}=1,32$; IC95\%: 1,19-1,47). Esse mesmo padrão, também foi observado para as outras formas de apoio social dos pais.

\section{Discussão}

$\mathrm{O}$ presente estudo mostrou que a prevalência de inatividade física em escolares de Macapá (AP) é preocupante, com $60 \%$ dos adolescentes não praticando pelo menos 300 minutos por semana em atividades físicas. Ainda, este estudo mostrou que aqueles adolescentes que pouco recebiam apoio familiar dos pais tinham mais chances de serem inativos, quando comparado aos seus pares.

A prevalência de inatividade física encontrada no presente estudo (60\%) foi a semelhante e corrobora com vários estudos encontrados pelo Brasil e no mundo $9,12,13,14,1925$. A maior prevalência de inatividade física no sexo feminino já era um resultado esperado. Dados da Pesquisa Nacional de Saúde do Escolar (PeNSE), realizado em escolares do ensino fundamental e médio, mostraram que mais da metade $(56,2 \%)$ dos adolescentes do sexo masculino foram classificados como fisicamente ativos, e que apenas $31,1 \%$ das adolescentes eram ativas ${ }^{1}$. Estudos conduzidos em outros países também têm encontrado o mesmo padrão na relação entre sexo e inatividade física em adolescentes. Revisão sistemática recente, identificou que em 22 dos 24 estudos analisados, as adolescentes foram consideradas fisicamente menos ativas do que seus pares do sexo masculino ${ }^{15}$.

A alta prevalência de inatividade física no presente estudo deve ser explicada dentro de um contexto sociocultural de Macapá, na qual há carência de espaços apropriados para a pratica de atividade física. Além do mais, um cenário local com déficit habitacional, ausência de ciclovias e a ilegalidades dos espaços para construções habitacionais situadas na maioria das vezes em áreas de ressacas (casas sobe pequenas vielas de rios) podem contribuir para este desfecho. Este contexto se contrapõe a um ambiente propício e saudável para se tornar fisicamente ativo, proporcionando um cenário que pode causar modificações negativas no comportamento dos indivíduos impactando diretamente na 
Tabela 3 - Prevalência de inatividade física de acordo com apoio social dos pais e regressão de Poisson para associação entre apoio social familiar e inatividade física em adolescentes da cidade de Macapá (Amapá), 2016, ( $\mathrm{n}=1.205$ ).

\begin{tabular}{|c|c|c|c|c|c|c|}
\hline Apoio social dos pais & $\mathrm{n}(\%)$ & $\begin{array}{c}\text { Fisicamente inativo } \\
\mathrm{n}(\%)\end{array}$ & $\begin{array}{l}\text { RP bruta } \\
\text { (IC95\%) }\end{array}$ & $\mathrm{p}$ & $\begin{array}{l}\text { RP ajustada } \\
\text { (IC95\%) }\end{array}$ & $\mathrm{p}$ \\
\hline Estimulam os filhos a praticarem atividade física & & & & $<0,001$ & & $<0,001$ \\
\hline Frequentemente/sempre & $471(39,1)$ & $228(48,4)$ & 1,00 & & 1,00 & \\
\hline Nunca/raramente & $734(60,9)$ & $498(68,9)$ & $1,40(1,26-1,56)$ & & $1,32(1,19-1,47)$ & \\
\hline Praticam atividade física com os filhos & & & & $<0,001$ & & $<0,001$ \\
\hline Frequentemente/sempre & $243(20,2)$ & $111(45,7)$ & 1,00 & & 1,00 & \\
\hline Nunca/raramente & $962(79,8)$ & $615(63,9)$ & $1,40(1,21-1,62)$ & & $1,36(1,18-1,57)$ & \\
\hline Transportam/disponibilizam transporte & & & & $<0,001$ & & $<0,001$ \\
\hline Frequentemente/sempre & $486(40,3)$ & $253(52,1)$ & 1,00 & & 1,00 & \\
\hline Nunca/raramente & $719(59,6)$ & $473(65,8)$ & $1,26(1,14-1,40)$ & & $1,22(1,10-1,35)$ & \\
\hline Assistem os filhos durante a atividade física & & & & $<0,001$ & & $<0,001$ \\
\hline Frequentemente/sempre & $265(22,0)$ & $127(47,9)$ & 1,00 & & 1,00 & \\
\hline Nunca/raramente & $940(78,0)$ & $599(63,7)$ & $1,33(1,16-1,52)$ & & $1,31(1,15-1,49)$ & \\
\hline Conversam com os filhos sobre atividade física & & & & $<0,001$ & & $<0,001$ \\
\hline Frequentemente/sempre & $457(37,9)$ & $242(53,0)$ & 1,00 & & 1,00 & \\
\hline Nunca/raramente & $748(62,1)$ & $484(64,7)$ & $1,22(1,10-1,35)$ & & $1,20(1,08-1,33)$ & \\
\hline Elogiam a prática das atividades físicas dos filhos & & & & $<0,001$ & & $<0,001$ \\
\hline Frequentemente/sempre & $419(34,8)$ & $202(48,2)$ & 1,00 & & 1,00 & \\
\hline Nunca/raramente & $786(65,2)$ & $524(66,7)$ & $1,38(1,24-1,54)$ & & $1,34(1,20-1,50)$ & \\
\hline
\end{tabular}

RP= Razão de prevalência; IC95\%= Intervalo de confiança de 95\%; *Teste do Qui-quadrado; ajuste para sexo, escolaridade do pai e ano no ensino médio.

percepção de qualidade de vida, segurança e saúde e no aumento da inatividade física ${ }^{16}$.

No presente estudo, os escolares do $1^{\circ}$ ano e $2^{\circ}$ anos do ensino médio tiveram maior prevalência de inatividade física comparados aos do $3^{\circ}$ ano. Torna-se importante considerar que não há indicativo plausível de que a existência da elevada carga de estudos no $3^{\circ}$ ano pode estabelecer níveis de inatividade física maior em relação aos demais anos do ensino médio. Porém, se torna preocupante o fato de os escolares ingressantes no ensino médio já entrarem com uma prevalência de inatividade elevada antes mesmo alcançar o período de dedicação aos estudos em razão dos processos seletivos e vestibulares se submetem no último ano do ensino médio.

Este estudo mostrou que a ausência de apoio dos pais dos pais para a prática de atividade física foi positivamente associada com maiores níveis de inatividade física. Pesquisas que tratam como exposição o apoio da família, tendo como desfecho a inatividade física na população jovem do Brasil passaram a ser recorrentes ${ }^{1,2,9}$. Porém, o presente estudo foi um dos primeiros com essa discussão em uma capital do Norte do Brasil.

De uma forma geral, observou-se que o falta de apoio dos pais esteve associado com maiores prevalências de inatividade física. Verificou-se que pais que nunca estimularam os filhos a fazer atividade física, que jamais praticaram em conjunto, nem transportavam ou disponibilizavam transporte, nunca assistiram os filhos a praticarem atividade física, muito menos conversavam sobre a temática ou até mesmo elogiaram os resultados alcançados e tiveram filhos mais inativos quando comparados aos pais que forneciam alguma forma de apoio social aos filhos.

Estudos prospectivos ${ }^{17} 24$ demonstraram relação inversa entre apoio social e declínio do nível de atividade física com o aumento da idade dos adolescentes ${ }^{10}$. Por exemplo, em estudo realizado em Pelotas adolescentes que tinham pais para incentivar atividade física organizada foram aqueles que, em média, apresentaram maior número de dias envolvidos em atividade física moderada comparados aos adolescentes que não tinham pais para incentivar a prática de exercício físico ${ }^{8}$.

Outro estudo nacional verificou a falta de companhia tanto para prática quanto para assistir os filhos em suas atividades físicas esteve associado de forma positiva com a inatividade física em adolescentes ${ }^{22}$. Quando a família não acompanha ou interage, assistindo ou praticando atividade física com os filhos, torna-se mais provável a ocorrência de desvalorização em uma possível mudança comportamental do adolescente, diminuindo a autoestima, tornando-os mais inseguros e com baixa confiança deixar de ser fisicamente inativo ${ }^{22}$. A ausência da companhia familiar encoraja o adolescente a ter menos interações sociais ${ }^{4}$, as quais podem favorecer um 
estilo de vida menos ativo fisicamente em função da desmotivação em realizar atividade física. Em estudos internacionais foi observado que pais que eram fisicamente mais inativos tendiam a fornecer menos apoio social para prática de atividade física dos seus filhos ${ }^{1718}$.

Vale a pena ressaltar que envolvimento e a supervisão dos pais nas atividades físicas, fornecimento de deslocamento por meio de transporte, aquisição de materiais e equipamentos para a prática do exercício físico ajudam os adolescentes a estabelecer uma vida fisicamente ativa, além de propiciar maior aproximação afetiva entre pais e filhos, estando às vezes com a carência dessa proximidade ${ }^{7}$. Verificou-se que não há estabelecimento de achados semelhantes na literatura quanto à influência dos pais na prática de atividade física dos filhos, porque nem sempre o apoio social dado pelos pais corresponde a vontade dos filhos quanto ao seu estilo de vida ${ }^{20}$. Em geral, os estudos com adolescentes têm identificado que há influência dos pais em diversos comportamentos relacionados à saúde, incluindo a prática de atividade física ${ }^{21}$.

Algumas limitações do estudo merecem consideradas na interpretação dos achados. $\mathrm{O}$ fato da exposição e desfecho terem sidos coletados no mesmo período não permite estabelecer uma relação causal entre apoio dos pais e inatividade física em adolescentes. Outra limitação, diz respeito a forma subjetiva como as variáveis de apoio dos pais e prática de atividade física foram coletadas. Entretanto, é pouco provável que esta forma de medida acrescente algum erro diferencial nas nossas medidas de exposição e desfecho, sugerindo que a medida de associação encontrada possa estar, de certa forma, subestimada. Apesar disso, os dados do presente estudo podem fornecer importantes informações, especialmente com possibilidade de extrapolação dos achados, exigindo certa cautela, para capitais da região Norte do Brasil.

Conclui-se que a proporção de escolares de 14 a 19 anos de idade fisicamente inativos em Macapá foi elevada, sobretudo no sexo feminino e nos estudantes do ensino médio ( $1^{\circ}$ e $2^{\circ}$ ano). Ainda, verificou-se que adolescentes cujo os pais proporcionam baixo o apoio social para a atividade física foi mais inativo que seus pares. Frente a este cenário, é necessário que o poder público nas esferas municipal, estadual e federal adote através de políticas públicas ações que possibilitem a população jovem ter a adoção de estilo de vida fisicamente ativo, bem como inseri-los em programas de atividade física sistematizadas para aumentar o apoio social dos pais e a criação de espaços adequados para a prática de atividade física.

\section{Conflito de interesses}

Os autores declaram não haver conflito de interesses.

\section{Contribuição dos autores}

Costa DJS participou da concepção inicial do estudo, coleta de dados, análise estatística redação, revisão crítica do texto. Pureza DY contribuiu com a orientação, redação e revisão crítica do manuscrito. Mielke GI contribuiu com revisão crítica e análise estatística.

\section{Referências}

1. Brasil, Ministério da Saúde, Instituto Brasileiro de Geografia e Estatística. Pesquisa Nacional de Saúde do Escolar. Rio de Janeiro: Ministério da Saúde, Instituto Brasileiro de Geografia e Estatística; 2009. Disponível em: https://biblioteca.ibge. gov.br/visualizacao/livros/liv97870.pdf.

2. Alves CF, Silva RC, Assis AM, Souza CO, Pinto EJ, Frainer DE. Fatores associados à inatividade física em adolescentes de 10-14 anos de idade. Rev Bras de Epid. 2012;15(4):858-70.

3. Farias Júnior JC. Prevalência e fatores de influência para inatividade física em adolescentes. Rev Bras de Ciên e Mov. 2006;14(2):57-64.

4. Prado CV, Lima AV, Fermino RC, Anez CR, Reis RS. Apoio social e prática de atividade física em adolescentes da rede pública de ensino: qual a importância da família e dos amigos? Cad Saude Publica. 2014;30(4):827-38.

5. Vrazel JE, Saunders RP, Wilcox S. An overview and proposed framework of social-enviromental influences on the physicalactivity behavior of women. Am J Health Promot. 2008;23:2-12.

6. Van der horst K, Paw MJ, Twisk JW, Van Mechelen W. A brief review on correlates of physical activity and sedentariness in youth. Med Sci Sports Exerc. 2007;39(8):1241-50.

7. Sallis J, Taylor WC, Dowda M, Freedson PS, Pate RR. Correlates of vigorous physical activity for children in grades 1 through 12: comparing parent-reported and objectively measured physical activity. Ped Exer Sci. 2002;14:30-44.

8. Peixoto MB, Prática de Atividade Física e Suporte Social em Adolescentes: Um estudo de base populacional [Dissertação de Mestrado]. Pelotas: Universidade Federal de Pelotas, Pelotas; 2012.

9. Silva KS, Nahas MV, Peres KG, Lopes AS. Fatores associados à atividade física, comportamento sedentário e participação na Educação Física em estudantes do Ensino Médio em Santa Catarina, Brasil. Cad Saude Publica. 2009; 25(10):2187-00.

10. Farias Júnior JC, Lopes AS, Mota J, Hallal PC. Prática de atividade física e fatores associados em adolescentes do Nordeste do Brasil. Rev Saude Publica. 2012;46(3):505-15

11. Associação Brasileira de Empresas de Pesquisa. Critério de Classificação Econômica Brasil 2010. ABEP; 2010. Dados do acesso.

12. Ceschini FB, Figueira Jr A. Barreiras e determinantes para a prática de atividade física em adolescentes. Revista Brasileira Ciência Movimento. 2007;15(1):29-36.

13. Hallal PC, Bertoldi AD, Gonçalves H, Victora CG. Prevalência de sedentarismo e fatores associados em adolescentes de 10-12 anos de idade. Cad Saude Publica, 2006;6(22):1277-87.

14. Telama R, Yang X, Viikari J, Valimaki I, Wanne O, Raitakari O. Physical activity from childhood to adulthood: a 21-years tracking study. Am J Prev Med. 2005;28(3):267-73. 
15. Seabra AF, Mendonça DM, Thomis MA. Anjos LA, Maia JA. Determinantes biológicos e socioculturais associados à prática de atividade física de adolescentes. Cadernos de Saúde Pub. 2008;24:721-36.

16. Plugliese J, Tinsley B. Parental socialization of child and adolescent physical activity: a meta-analysis. Jour Psych. 2007;21:331-43.

17. Dowda M, Dishman RD, Pfeiffer KA, Pate RR. Family support for physical activity in girls from 8 th to 12 th grade in South Carolina. Prev Med. 2007;44:153-9.

18. Fernandes RA, Chistofaro DG, Milanez VF, Casonatto J, Cardoso JR, Ronque ER . et.al. Atividade física: prevalência, fatores relacionados e associação entre pais e filhos. Rev Paul de Ped. 2011;29(1):54-9.

19. Loch M. Indicadores de Saúde e do Estilo de Vida de Adolescentes Escolares Residentes em Municípios Grandes, Médios e Pequenos de Santa Catarina, Brasil. Rev Bras de Ciênc e Mov. 2007;15(3):7-15.

20. Newman K, Harrison L, Dashiff C. Davies S. Relationships between parenting styles and risk behaviors in adolescent health: an integrative literature review. Rev Latino-Am Enfer. 2008:16(1):142-50
21. Souza, CA, Rech CR, Sarabia TT, Anez, CR. Reis RS. Autoeficácia e atividade física em adolescentes de Curitiba, Paraná, Brasil. Cad de Saúde Pub. 2013;29(10):2039-48.

22. Santos MS, Hino AAF, Reis RS, Añez CRR. Prevalência de barreiras para a prática de atividade física em adolescentes. Rev Bras Epidemiol. 2010;13:94-104

23. Bandura A. Health promotion by social cognitive means. Health Educ Behav 2004;31:143-64.

24. Dishman RK, Saunders RP, Molt RW, Dowda M, Pate RR. Self-efficacy moderates the relation between declines in physical activity and perceived social support in high school girls. J Pediatr Psychol. 2009;34:441-51.

25. Mello, MRI. O nível de atividade física em adolescentes escolares do ensino público da cidade de Franca-SP [Dissertação de Mestrado]. São Paulo: Universidade de Franca, 2011.

\section{Como citar este artigo:}

Costa DJS, Pureza DY, Mielke GI. Prevalência de inatividade física e apoio social dos pais em adolescentes de Macapá-AP. Rev Bras Ati Fis Saúde. 2017;22(6):533-39 DOI: 10.12820/rbafs.v.22n6p533-39.

Recebido: 10/03/2016

Aprovado: 27/10/2017 